\title{
Efficiency at maximum power of minimally nonlinear irreversible heat engines
}

\author{
Y. IZUMIDA and K. OKuDA \\ Division of Physics, Hokkaido University - Sapporo, 060-0810, Japan
}

PACS 05.70.Ln - Nonequilibrium and irreversible thermodynamics

\begin{abstract}
We propose the minimally nonlinear irreversible heat engine as a new general theoretical model to study the efficiency at the maximum power $\eta^{*}$ of heat engines operating between the hot heat reservoir at the temperature $T_{h}$ and the cold one at $T_{c}\left(T_{c} \leq T_{h}\right)$. Our model is based on the extended Onsager relations with a new nonlinear term meaning the power dissipation. In this model, we show that $\eta^{*}$ is bounded from the upper side by a function of the Carnot efficiency $\eta_{C} \equiv 1-T_{c} / T_{h}$ as $\eta^{*} \leq \eta_{C} /\left(2-\eta_{C}\right)$. We demonstrate the validity of our theory by showing that the low-dissipation Carnot engine can easily be described by our theory.
\end{abstract}

Introduction. - Facing with the recent worldwide problems such as the global warming and the depletion of ' energy resources, we have been urged to a low-carbon sustainable society. Demands for more efficient and greener heat engines have rapidly been rising since heat engines ' convert heat energy into useful work by utilizing only temperature difference, which is abundant in the earth's environment: geothermal power generation, solar thermal power generation, etc. might be promising candidates, for - example. To evaluate and control performance of heat engines, it must be important to know the upper bound of the energy conversion efficiency of them. The efficiency $\eta$ for a heat engine is defined as $\eta \equiv W / Q_{h}$, where $Q_{h}$ and $W$ denote the heat transferred from the hot heat reservoir ' at the temperature $T_{h}$ and the work output, respectively. Defining $Q_{c}$ as the heat transferred from the cold heat 'reservoir at the temperature $T_{c}\left(\leq T_{h}\right)$, we can express $W$ as $W \equiv Q_{h}+Q_{c}$. Thermodynamics tells us that $\eta$ is 'bounded from the upper side as

$$
\eta \leq 1-\frac{T_{c}}{T_{h}} \equiv \eta_{C},
$$

where $\eta_{C}$ denotes the Carnot efficiency and the equality holds only when the heat engine is infinitely slowly (quasistatically) operated to satisfy reversibility. Because the heat engine realizing the Carnot efficiency takes infinte time to output a finite amount of work, its power (work output per unit time) is absolutely 0 and thus it is of no practical use. Motivated by this fact, Curzon and Ahlborn [1] proposed a phenomenological finite-time Carnot cycle model and derived that the efficiency at the maximum power $\eta^{*}$ of their model is given by an appealing expression as

$$
\eta^{*}=1-\sqrt{\frac{T_{c}}{T_{h}}} \equiv \eta_{C A},
$$

which reminds us of the Carnot efficiency. Historically and strictly speaking, the formula $1-\sqrt{T_{c} / T_{h}}$ itself was derived by others 2, 3, more previously than [1. But it is usually called the Curzon-Ahlborn (CA) efficiency. We also call it the CA efficiency here in accord with the custom. The paper by Curzon and Ahlborn triggered subsequent studies on the efficiency at the maximum power of various heat engine models [4 37 . Among recent studies on the $\mathrm{CA}$ efficiency, it is an important progress that Van den Broeck 12 proved that the CA efficiency $\Delta T /(2 T)=\eta_{C A}+O\left(\Delta T^{2}\right)$ is the upper bound of the efficiency at the maximum power for the heat engines working in the linear response regime. Here, we have defined the temperature difference $\Delta T \equiv T_{h}-T_{c}$, which is assumed to be small, and the averaged temperature $T \equiv\left(T_{h}+T_{c}\right) / 2$, respectively. Those heat engines working in the linear response regime, which we call the linear irreversible heat engines, are described by the following Onsager relations [38, 39]:

$$
\begin{aligned}
& J_{1}=L_{11} X_{1}+L_{12} X_{2}, \\
& J_{2}=L_{21} X_{1}+L_{22} X_{2},
\end{aligned}
$$

where $X_{1} \equiv F / T_{c} \simeq F / T$ with an external force $F, J_{1} \equiv \dot{x}$ with the conjugate variable $x$ of $F, X_{2} \equiv 1 / T_{c}-1 / T_{h} \simeq$ $\Delta T / T^{2}, J_{2} \equiv \dot{Q}_{h}$ and $L_{i j}$ 's are the Onsager coefficients 
with the reciprocity $L_{12}=L_{21}$. The dot denotes the quantity per unit time. Regarding $X_{1}$ as the control parameter for the maximization of the power $P=-F \dot{x}=-J_{1} X_{1} T$, we can see that the maximum power $P^{*}$ and the efficiency at the maximum power $\eta^{*}$ of the linear irreversible heat engines described by eqs. (3) and (4) are given by

$$
\begin{aligned}
& P^{*}=\frac{q^{2} L_{22} \Delta T^{2}}{4 T^{3}}, \\
& \eta^{*}=\frac{\Delta T}{2 T} \frac{q^{2}}{2-q^{2}},
\end{aligned}
$$

respectively 12, where $q$ is called the coupling strength parameter and is defined as

$$
q \equiv \frac{L_{12}}{\sqrt{L_{11} L_{22}}} .
$$

Since the positivity of the entropy production rate $\dot{\sigma} \equiv$ $J_{1} X_{1}+J_{2} X_{2}$, which is a quadratic form of the thermodynamic forces, restricts the Onsager coefficients $L_{i j}$ 's to

$$
L_{11} \geq 0, L_{22} \geq 0, L_{11} L_{22}-L_{12} L_{21} \geq 0,
$$

$q$ should take $-1 \leq q \leq 1$. Thus $\eta^{*}$ in eq. (6) takes the upper bound $\Delta T /(2 T)$ which is equal to the CA efficiency up to the linear order of $\Delta T$, when the tight-coupling condition

$$
|q|=\left|\frac{L_{12}}{\sqrt{L_{11} L_{22}}}\right|=1
$$

holds. This condition is equivalent to saying that the two thermodynamic fluxes become proportional as $J_{2} \propto J_{1}$. Due to the generality of the theory, the study by Van den Broeck renewed the interests in the CA efficiency and inspired the studies on the efficiency at the maximum power of various heat engine models: it has been indeed shown that $\eta^{*}$ of many heat engine models, ranging from the steady-state brownian motors [13, 17, 18 to the finite-time Carnot cycles [30, 31, is given by eq. (6) in the linear response regime. Even when the system begins to enter the nonlinear response regime, it is often observed that $\eta^{*}$ agrees with the CA efficiency up to the quadratic order of $\Delta T$ as $\eta^{*}=\Delta T /(2 T)+\Delta T^{2} /\left(8 T^{2}\right)=$ $\eta_{C A}+O\left(\Delta T^{3}\right)[24,27,34,35$. This fact was firstly observed in 24] and proposed as a conjecture in 25. Later, it was proved to be a precise result for the system which satisfies the left-right symmetry condition in addition to the tight-coupling condition in 27]. However, it is also shown, for example in $\left[19,20,24,29,34,35\right.$, that $\eta^{*}$ can exceed the CA efficiency in the nonlinear response regime, when those conditions do not hold. Therefore the CA efficiency is no longer the upper bound of $\eta^{*}$ for nonlinear irreversible heat engines and we need to construct a general theory to determine the upper bound of $\eta^{*}$ for them.

In this paper, we propose the minimally nonlinear irreversible heat engine described by the extended Onsager relations, where a new nonlinear term $-\gamma_{h} J_{1}{ }^{2}$ meaning the power dissipation is added to eq. (4) (see eq. (12)). The addition of this new nonlinear term can be seen as a natural extension of the linear irreversible heat engine and we formulate $\eta^{*}$ of such nonlinear irreversible heat engines. Our new formula eq. (20) contains the coupling strength parameter $q$ and a parameter $\gamma_{c} / \gamma_{h}$, where $\gamma_{c}$ and $\gamma_{h}$ denote the degree of dissipation to the cold and hot heat reservoirs, respectively. We show that our $\eta^{*}$ is bounded from the upper side by a function of the Carnot efficiency $\eta_{C}$ as $\eta^{*} \leq \eta_{+}$, where $\eta_{+} \equiv \eta_{C} /\left(2-\eta_{C}\right)$. Remarkably this $\eta_{+}$was also mentioned in the previous studies on various finite-time heat engine models [24, 32, 35, 40, 41]. The generality of our theory allows us to unify these previous results and explain the universality of $\eta_{+}$. For a demonstration of the validity of our theory, we show that a finitetime Carnot cycle model, called the low-dissipation Carnot engine [35, can be described by the extended Onsager relations.

Extended Onsager relations. - Let us consider that a general heat engine is working between the hot and the cold heat reservoirs with the temperature difference $\Delta T=T_{h}-T_{c}$. Heat engines are generally classified into two types: steady-state heat engines and cyclic heat engines. Steady-state heat engines literally work in a steady state since an external force applied on the heat engines is time-independent and the hot and cold heat reservoirs contact with the heat engines simultaneously. Cyclic heat engines, in contrast, work cyclically in a time-dependent way such that the hot and the cold heat reservoirs contact with the heat engines alternately, not simultaneously. Our theory below can treat both types of heat engines in a unified manner. We can generally write the total entropy production rate $\dot{\sigma}$ of the heat engine as the entropy increase rate of the heat reservoirs as

$$
\dot{\sigma}=-\frac{\dot{Q}_{h}}{T_{h}}-\frac{\dot{Q}_{c}}{T_{c}}=-\frac{\dot{W}}{T_{c}}+\dot{Q}_{h}\left(\frac{1}{T_{c}}-\frac{1}{T_{h}}\right),
$$

where we do not need to consider the entropy increase inside the heat engine since the heat engine itself is always in a steady state or comes back to the original state after one cycle. Note that the dot denotes the quantity per unit time for steady-state heat engines and the quantity divided by the one-cycle period $\tau_{c y c}$ for cyclic heat engines. The power $P \equiv \dot{W}$ is expressed as $P=-F \dot{x}$ for steadystate heat engines where the time-independent external force $F$ is acting on its conjugate variable $x$, and as $P=$ $W / \tau_{\text {cyc }}$ for cyclic heat engines. From the decomposition $\dot{\sigma} \equiv J_{1} X_{1}+J_{2} X_{2}$, we can define the thermodynamic force $X_{1} \equiv F / T_{c}$ and its conjugate thermodynamic flux $J_{1} \equiv \dot{x}$ for steady-state heat engines as well as $X_{1} \equiv-W / T_{c}$ and $J_{1} \equiv 1 / \tau_{c y c}$ for cyclic heat engines. We can also define the other thermodynamic force $X_{2} \equiv 1 / T_{c}-1 / T_{h}$ and its conjugate thermodynamic flux $J_{2} \equiv \dot{Q}_{h}$ for both types of heat engines. By using these thermodynamic fluxes and forces, the power $P$ is rewritten as $P=-J_{1} X_{1} T_{c}$. We assume that these thermodynamic fluxes and forces satisfy 


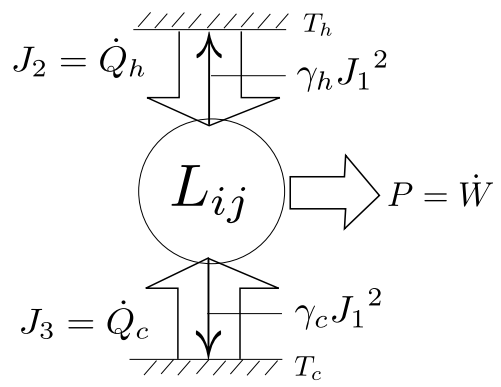

Fig. 1: Schematic illustration of the minimally nonlinear irreversible heat engine described by eqs. (14) and (15).

the following extended Onsager relations:

$$
\begin{aligned}
& J_{1}=L_{11} X_{1}+L_{12} X_{2}, \\
& J_{2}=L_{21} X_{1}+L_{22} X_{2}-\gamma_{h} J_{1}{ }^{2},
\end{aligned}
$$

where the features of the Onsager coefficients $L_{i j}$ 's in eqs. (3) and (41) are assumed to hold also in eqs. (11) and (12). In eq. (12), the new nonlinear term $-\gamma_{h} J_{1}^{2}$ is introduced into the standard Onsager relation eq. (4) and $\gamma_{h}$ is assumed to be a positive constant as $\gamma_{h}>0$. We also assume that no other higher nonlinear terms arise in eqs. (11) and (12) by considering that the coefficients of higher nonlinear terms are too small to have effective contributions to $\dot{\sigma}$ compared to $-\gamma_{h} J_{1}{ }^{2}$, as will be seen in eq. (18). In our model, we also assume that $X_{1}$ and $X_{2}$ are not restricted to small values, unlike in the linear irreversible heat engine described by eqs. (3) and (4), where the limit of $X_{1} \rightarrow 0$ and $X_{2} \rightarrow 0$ is taken.

Here we introduce the heat flux from the cold heat reservoir

$$
\dot{Q}_{c}=P-\dot{Q}_{h}=-J_{1} X_{1} T_{c}-J_{2} \equiv J_{3} .
$$

Then we can rewrite the heat fluxes eqs. (12) and (13) into more suggestive and symmetrical forms as (see fig. 10)

$$
\begin{aligned}
& J_{2}=\frac{L_{21}}{L_{11}} J_{1}+L_{22}\left(1-q^{2}\right) X_{2}-\gamma_{h} J_{1}{ }^{2}, \\
& J_{3}=-\frac{L_{21} T_{c}}{L_{11} T_{h}} J_{1}-L_{22}\left(1-q^{2}\right) X_{2}-\gamma_{c} J_{1}{ }^{2},
\end{aligned}
$$

where $q$ is defined in eq. (7) and $\gamma_{c}$ is defined as

$$
\gamma_{c} \equiv \frac{T_{c}}{L_{11}}-\gamma_{h}
$$

We assume $\gamma_{c}$ to be a positive constant as $\gamma_{c}>0$. We can use eqs. (14) and (15) to describe the heat engines instead of eqs. (11) and (12), regarding $J_{1}$ as the control parameter of the heat engines instead of $X_{1}$ since $J_{1}$ and $X_{1}$ are uniquely related through eq. (11) when $X_{2}$ is fixed. We call the heat engines described by eqs. (14) and (15) (or the extended Onsager relations eqs. (11) and (12)) the minimally nonlinear irreversible heat engines. The term "minimally" implies that we take into account only $-\gamma_{h}{J_{1}}^{2}$ and $-\gamma_{c} J_{1}^{2}$ as the nonlinear terms. As we explain below, those terms will turn out to be the inevitable power dissipations accompanied by the finite-time motion of the heat engines.

The power $P=-J_{1} X_{1} T_{c}=J_{2}+J_{3}$ can be rewritten as

$$
P=\frac{L_{21}}{L_{11}} \eta_{C} J_{1}-\frac{T_{c}}{L_{11}} J_{1}^{2},
$$

by adding eqs. (14) and (15). Here we immediately notice that the second terms in eqs. (14) and (15) do not contribute to eq. (17) at all. They mean just the direct heat transfer from the hot heat reservoir to the cold one, which arises in the case of the non-tight coupling condition $|q| \neq 1$. Note that similar decomposition of the power like eq. (17) is also given in 32. The first term in eq. (17) is proportional to $\Delta T$ through $\eta_{C}$, meaning the power generation due to the temperature difference. On the other hand, the second term in eq. (17) remains nonzero even when $\Delta T=0$ and means the power dissipation which should necessarily be consumed once the heat engine moves at a finite rate $\left(J_{1} \neq 0\right)$ regardless of how small $J_{1}$ is. The power dissipation results in the increase of the total internal energy of the heat reservoirs. When $\Delta T=0$, this is nothing but the effect of Joule heating, which is seen if we can consider that $T_{c} / L_{11}$ and $J_{1}$ in eq. (17) correspond to resistance and electric current, respectively.

To clarify the physical meaning of each term in eqs. (14) and (15) in more detail, we rewrite the total entropy production rate $\dot{\sigma}=-\dot{Q}_{h} / T_{h}-\dot{Q}_{c} / T_{c}=-J_{2} / T_{h}-J_{3} / T_{c}$ as

$$
\dot{\sigma}=L_{22}\left(1-q^{2}\right) X_{2}^{2}+\frac{J_{1}{ }^{2}}{L_{11}}-\gamma_{h} J_{1}^{2} X_{2},
$$

by using eqs. (14) and (15). The first term means the entropy increase rate of the heat reservoirs due to the direct heat transfer. The second term comes from the inevitable work consumption due to the finite-time operation. The third term in eq. (18) arises due to the presence of $-\gamma_{h} J_{1}{ }^{2}$ in eq. (12). In the case of the linear irreversible heat engine described by eqs. (3) and (44), the third term is suppressed and the non-negativity of $\dot{\sigma}$ restricts the Onsager coefficients $L_{i j}$ 's to eq. (8). Even in our model, we assume that the restriction eq. (8) still holds although $X_{1}$ and $X_{2}$ are not restricted to small values. Then the non-negativity of eq. (18) is always guaranteed since it is rewritten as $\dot{\sigma}=L_{22}\left(1-q^{2}\right) X_{2}{ }^{2}+\left(\gamma_{h} / T_{h}+\gamma_{c} / T_{c}\right) J_{1}{ }^{2}$ by using eq. (16): the first term is always non-negative due to eq. (8) and the second one is also always non-negative due to the assumptions $\gamma_{h}>0$ and $\gamma_{c}>0$.

Efficiency at maximum power. - We consider the efficiency of the heat engine $\eta=W / Q_{h}=P / \dot{Q}_{h}=P / J_{2}$, where $P$ and $J_{2}$ are given in eqs. (17) and (14), respectively. When $X_{2}$ and $L_{i j}$ 's are given, the maximum power is realized at $J_{1}=L_{12} X_{2} / 2$ as a solution of $\partial P / \partial J_{1}=0$ 
and then we obtain the maximum power $P^{*}$ and the efficiency at the maximum power $\eta^{*}$ as

$$
\begin{aligned}
P^{*} & =\frac{q^{2} L_{22} \Delta T^{2}}{4 T_{h}{ }^{2} T_{c}}, \\
\eta^{*} & =\frac{\eta_{C}}{2} \frac{q^{2}}{2-q^{2}\left(1+\eta_{C} /\left(2\left(1+\gamma_{c} / \gamma_{h}\right)\right)\right)},
\end{aligned}
$$

respectively. The formula eq. (20) is the main result of this paper. We notice that it includes the formula eq. (6) of the linear irreversible heat engine as the linear term of $\eta_{C} \simeq \Delta T / T$ in the limit of $\Delta T \rightarrow 0$. We also notice that eq. (20) has the lower bound $\eta_{-}^{q}$ and the upper bound $\eta_{+}^{q}$ at a fixed $q$ as

$$
\eta_{-}^{q} \equiv \frac{\eta_{C}}{2} \frac{q^{2}}{2-q^{2}} \leq \eta^{*} \leq \frac{\eta_{C}}{2} \frac{q^{2}}{2-q^{2}\left(1+\eta_{C} / 2\right)} \equiv \eta_{+}^{q},
$$

by taking the asymmetrical dissipation limits $\gamma_{c} / \gamma_{h} \rightarrow$ $\infty$ and $\gamma_{c} / \gamma_{h} \rightarrow 0$, respectively. Moreover $\eta_{+}^{q}$ takes the maximum value

$$
\eta_{+}^{q} \leq \frac{\eta_{C}}{2-\eta_{C}} \equiv \eta_{+},
$$

when the tight-coupling condition $|q|=1$ is satisfied in $\eta_{+}^{q}$. Therefore this $\eta_{+}$is the upper bound of $\eta^{*}$ for the minimally nonlinear irreversible heat engines.

We note that $\eta_{+}$has also been found in the previous studies on the efficiency at the maximum power: in a finite-time heat engine model [40], where the heat fluxes are assumed to obey a specific conduction law, $\eta_{+}$arises as a limiting case. In a Feynman ratchet model [41, $\eta_{+}$ has been obtained as Feynman efficiency under the no heat leak condition between the heat reservoirs. In a few finitetime Carnot cycle models 24, 34, 35, $\eta_{+}$has been found as the upper bound of $\eta^{*}$ in the asymmetrical dissipation limit. Finally in 32] (see also [33]), $\eta_{+}$has been proved to be the upper bound of $\eta^{*}$ in the asymmetrical dissipation limit in a general and model-independent way. But the proof in 32 is limited to the case of stochastic steadystate heat engines. Our theory can be applicable to cyclic heat engines as well and unify these previous results.

Here we stress physical importance of $\eta_{+}$: if the efficiency at the maximum power of a finite-time heat engine exceeds $\eta_{+}$, it implies that the heat engine works under higher degree of nonequilibrium. In fact, we can see that $\eta^{*}$ of the finite-time Carnot cycle model of ideal gas reported in [28,29] exceeds $\eta_{+}$due to the higher nonequilibrium effect [42. Therefore $\eta_{+}$could be a criterion for determining the degree of nonequilibrium of finite-time heat engines.

Example: low-dissipation Carnot engine. - For a demonstration of the validity of our theory, we show that the low-dissipation Carnot engine [35] is described by the extended Onsager relations eqs. (11) and (12). Here the low-dissipation Carnot engine is a heat engine model proposed as a finite-time extension of the quasistatic Carnot cycle. It assumes the specific form of the heats transferred from the heat reservoirs during the isothermal processes as

$$
\begin{gathered}
Q_{h}=T_{h} \Delta S-\frac{T_{h} \Sigma_{h}}{\tau_{h}}+\cdots, \\
Q_{c}=-T_{c} \Delta S-\frac{T_{c} \Sigma_{c}}{\tau_{c}}+\cdots,
\end{gathered}
$$

where $\Delta S$ is the quasistatic entropy change inside the heat engine during the isothermal process in contact with the hot heat reservoir, $\tau_{h}$ and $\tau_{c}$ are the durations during the isothermal processes in contact with the hot heat reservoir and the cold one, respectively, and $\Sigma_{h}$ and $\Sigma_{c}$ are positive constants. We consider that the constants $\Sigma_{h}$ and $\Sigma_{c}$ contain the details how the engine deviates from the quasistatic limit. The assumption eqs. (23) and (24) means that the lowest deviation from the quasistatic heat should be proportional to the inverse of the duration. In a stochastic finite-time Carnot cycle model 24] analyzed by the Fokker-Planck equation, such a $\tau^{-1}$ term indeed arises [cf. eq. (16) in 24]]. In a finite-time Carnot cycle model of ideal gas analyzed by the molecular kinetic theory [28, it is also confirmed that the lowest deviation is proportional to $\tau^{-1}$ [cf. eq. (11) in [28]. Finally such a $\tau^{-1}$ term also arises in a quantum dot Carnot engine model 34 analyzed by the master equation approach [cf. eq. (28) in [34]]. Therefore the assumption of the specific form of the heats eqs. (23) and (24) has microscopically been justified in these models. Additionally, we neglect higher order terms such as $O\left(\tau^{-2}\right)$ in eqs. (23) and (24) in this low-dissipation approximation.

We can express the power $P=\left(Q_{h}+Q_{c}\right) /\left(\tau_{h}+\tau_{c}\right)$ of this engine as $P=\left(\Delta T \Delta S-T_{h} \Sigma_{h} / \tau_{h}-T_{c} \Sigma_{c} / \tau_{c}\right) /\left(\tau_{h}+\tau_{c}\right)$ by using eqs. (23) and (24). Maximizing this power by the durations $\tau_{h}$ and $\tau_{c}$ as $\partial P / \partial \tau_{h}=\partial P / \partial \tau_{c}=0$, we find the physically relevant solutions as

$$
\begin{gathered}
\tau_{h}=\frac{2 T_{h} \Sigma_{h}}{\left(T_{h}-T_{c}\right) \Delta S}\left(1+\sqrt{\frac{T_{c} \Sigma_{c}}{T_{h} \Sigma_{h}}}\right) \equiv \tau_{h}{ }^{*}, \\
\tau_{c}=\frac{2 T_{c} \Sigma_{c}}{\left(T_{h}-T_{c}\right) \Delta S}\left(1+\sqrt{\frac{T_{h} \Sigma_{h}}{T_{c} \Sigma_{c}}}\right) \equiv \tau_{c}{ }^{*} .
\end{gathered}
$$

Then by using the definition $\eta=\left(Q_{h}+Q_{c}\right) / Q_{h}$ and eqs. (23), (24), (25) and (26), we can obtain the efficiency at the maximum power $\eta^{*}$ as

$$
\eta^{*}=\frac{\eta_{C}\left(1+\sqrt{\frac{T_{c} \Sigma_{c}}{T_{h} \Sigma_{h}}}\right)}{\left(1+\sqrt{\frac{T_{c} \Sigma_{c}}{T_{h} \Sigma_{h}}}\right)^{2}+\left(1-\frac{\Sigma_{c}}{\Sigma_{h}}\right) \frac{T_{c}}{T_{h}}} .
$$

We can easily notice that eq. (27) is bounded from the lower side and the upper side as

$$
\frac{\eta_{C}}{2} \leq \eta^{*} \leq \frac{\eta_{C}}{2-\eta_{C}}
$$

by taking the asymmetrical dissipation limits $\Sigma_{c} / \Sigma_{h} \rightarrow \infty$ and $\Sigma_{c} / \Sigma_{h} \rightarrow 0$, respectively 35] (see also 34 for the 
derivation of these bounds in a quantum dot Carnot engine model). In 35, it is stated that observed efficiencies of various actual power plants tend to locate between these two bounds. It is also interesting to see that the same bounds were derived in a different finite-time heat engine model based on a specific heat conduction law [40]. By comparing eq. (28) with eq. (21), we may consider that the tight-coupling condition $|q|=1$ holds in this lowdissipation Carnot engine. We can prove it by writing the extended Onsager relations of this engine explicitly as follows.

First, we consider the total entropy production rate

$$
\begin{aligned}
\dot{\sigma} & =-\frac{\dot{Q}_{h}}{T_{h}}-\frac{\dot{Q}_{c}}{T_{c}}=-\frac{\dot{W}}{T_{c}}+\dot{Q}_{h}\left(\frac{1}{T_{c}}-\frac{1}{T_{h}}\right) \\
& =-\frac{W}{T_{c}(\alpha+1) \tau_{h}}+\dot{Q}_{h}\left(\frac{1}{T_{c}}-\frac{1}{T_{h}}\right),
\end{aligned}
$$

where we have defined the parameter $\alpha$ as $\alpha \equiv \tau_{c} / \tau_{h}$ and the dot denotes the quantity divided by the one-cycle pe$\operatorname{riod} \tau_{c y c}=\tau_{h}+\tau_{c}=(\alpha+1) \tau_{h}$. From the decomposition $\dot{\sigma}=J_{1} X_{1}+J_{2} X_{2}$, we can define the thermodynamic forces $X_{1} \equiv-W / T_{c}, X_{2} \equiv 1 / T_{c}-1 / T_{h}$ and their corresponding thermodynamic fluxes $J_{1} \equiv 1 /\left((\alpha+1) \tau_{h}\right), J_{2} \equiv \dot{Q}_{h}$. Using eqs. (23), (24) and the definitions of the thermodynamic forces and fluxes, we can easily calculate the Onsager coefficients $L_{i j}$ 's and the constant $\gamma_{h}$ of this low-dissipation Carnot engine as

$$
\begin{aligned}
& L_{11}=\frac{T_{c}}{\left(T_{h} \Sigma_{h}+T_{c} \Sigma_{c} / \alpha\right)(\alpha+1)}, \\
& L_{12}=\frac{T_{h} T_{c} \Delta S}{\left(T_{h} \Sigma_{h}+T_{c} \Sigma_{c} / \alpha\right)(\alpha+1)}, \\
& L_{21}=\frac{T_{h} T_{c} \Delta S}{\left(T_{h} \Sigma_{h}+T_{c} \Sigma_{c} / \alpha\right)(\alpha+1)}, \\
& L_{22}=\frac{T_{h}{ }^{2} T_{c} \Delta S^{2}}{\left(T_{h} \Sigma_{h}+T_{c} \Sigma_{c} / \alpha\right)(\alpha+1)}, \\
& \gamma_{h}=T_{h} \Sigma_{h}(\alpha+1),
\end{aligned}
$$

respectively. $\gamma_{c}$ is also given by

$$
\gamma_{c}=\frac{T_{c} \Sigma_{c}(\alpha+1)}{\alpha}
$$

by using eqs. (30), (34) and (16). We notice that the reciprocity $L_{12}=L_{21}$ surely holds from eqs. (31) and (32). Moreover we can confirm the tight-coupling condition $|q|=\left|L_{12} / \sqrt{L_{11} L_{22}}\right|=1$ from eqs. (30), (31) and (33) as expected. We can obtain $\eta^{*}$ of the low-dissipation Carnot engine as

$$
\eta^{*}=\frac{\eta_{C}}{2} \frac{1}{2-\left(1+\eta_{C} /\left(2\left(1+\frac{T_{c} \Sigma_{c}}{\alpha T_{h} \Sigma_{h}}\right)\right)\right)},
$$

by substituting $|q|=1$, eqs. (34) and (35) into eq. (20). We can also obtain $P^{*}$ as

$$
P^{*}=\frac{\Delta S^{2} \Delta T^{2}}{4\left(T_{h} \Sigma_{h}+T_{c} \Sigma_{c} / \alpha\right)(\alpha+1)}
$$

by substituting $|q|=1$, eq. (33) into eq. (19). However we notice that eq. (37) still contains the tunable parameter $\alpha$ and can further be maximized as $\partial P^{*}(\alpha) / \partial \alpha=0$, which reduces to $\alpha=\sqrt{T_{c} \Sigma_{c} /\left(T_{h} \Sigma_{h}\right)} \equiv \alpha^{*}$. From eqs. (25) and (26), we can see that $\alpha^{*}=\tau_{c}{ }^{*} / \tau_{h}{ }^{*}$ holds. Substituting this $\alpha^{*}$ into eq. (36), we finally reproduce eq. (27). Therefore we can conclude that the low-dissipation Carnot engine is exactly described by the extended Onsager relations. In other words, the inclusion of the power dissipation term $-\gamma_{h} J_{1}{ }^{2}$ into the Onsager relation as in eq. (12) is justified by this explicit example, whose assumptions eqs. (23) and (24) are consistent with the microscopically analyzed models [24,34.

Summary and discussion. - We proposed the minimally nonlinear irreversible heat engine described by the extended Onsager relations, where a new nonlinear term meaning the power dissipation is added to the heat flux from the hot heat reservoir in the standard Onsager relation and no other nonlinear terms are assumed to arise. Thus our model can be regarded as a natural and minimal extension of the linear irreversible heat engine. We formulated the efficiency at the maximum power $\eta^{*}$ of our model and showed that it is bounded from the upper side by $\eta_{C} /\left(2-\eta_{C}\right)$. This upper bound can be attained when the heat engine satisfies the tight-coupling condition $|q|=1$ and the asymmetrical dissipation limit $\gamma_{c} / \gamma_{h} \rightarrow 0$ is taken. As a demonstration of the validity of our theory, we explicitly wrote down the extended Onsager relations of the low-dissipation Carnot engine 35. and confirmed that it satisfies the tight-coupling condition $|q|=1$. Though the low-dissipation Carnot engine is an example of the cyclic heat engine, we should note that the power dissipation terms arise also in a few steady-state systems 43 45, where analytical calculations of the Onsager coefficients $L_{i j}$ 's, $\gamma_{h}$ and $\gamma_{c}$ are explicitly done based on a molecular kinetic theory. These calculations and the present example of the low-dissipation Carnot engine in this paper could support the validity of our theory, which treats the cyclic heat engines and the steady-state ones in the unified manner. It will be a future challenge to find the upper bound of the efficiency at the maximum power for more general irreversible heat engines with higher nonlinear terms beyond our model.

$$
* * *
$$

The authors thank N. Ito, K. Nemoto, M. Hoshina and S. Oono for helpful discussions. The authors also thank the Yukawa Institute for Theoretical Physics at Kyoto University. Discussions during the YITP workshop YITPW-10-16 on "YITP Workshop 2010: Physics of Nonequilibrium Systems -Fluctuation and Collective Behavior-" were useful to complete this work. YI acknowledges the financial support from a Grant-in-Aid for JSPS Fellows (Grant No. 22-2109). 


\section{REFERENCES}

[1] Curzon F. and Ahlborn B., Am. J. Phys., 43 (1975) 22.

[2] Yvon J., Proceedings of the International Conference on Peaceful Uses of Atomic Energy, 2 (United Nations Publications, Geneva, 1955) 337.

[3] Novikov I. I., J. Nuclear Energy, 7 (1958) 125.

[4] Rubin M. H., Phys. Rev. A, 19 (1979) 1272.

[5] Rubin M. H., Phys. Rev. A, 19 (1979) 1277.

[6] Landsberg P. T. and Leff H., J. Phys. A., 22 (1989) 4019.

[7] Gordon J. M., Am. J. Phys., 57 (1989) 1136.

[8] Kosloff R., J. Chem. Phys., 80 (1984) 1625.

[9] Benjamin A., J. Appl. Phys., 79 (1996) 1191.

[10] Asfaw M. and Bekele M., Eur. Phys. J. B, 38 (2004) 457.

[11] Asfaw M. and Bekele M., Phys. Rev. E., 72 (2005) 056109.

[12] Van den Broeck C., Phys. Rev. Lett., 95 (2005) 190602.

[13] Gomez-Marin A. and Sancho J. M., Phys. Rev. E, 74 (2006) 062102.

[14] Jiménez de Cisneros B. and Calvo Hernández A., Phys. Rev. Lett., 98 (2007) 130602.

[15] Jiménez de Cisneros B. and Calvo Hernández A., Phys. Rev. E, 77 (2008) 041127.

[16] Benjamin R. and Kawai R., Phys. Rev. E, 77 (2008) 051132 .

[17] Benjamin R., arXiv: 0907.0829v1.

[18] Gao T., Zhang Y. and Chen J., Chinese Phys. B., 18 (2009) 3279.

[19] Birjukov J., Jahnke T. and Mahler G., Eur. Phys. J. $B, 64$ (2008) 105.

[20] Allahverdyan A. E., Johal R. S. and Mahler G., Phys. Rev. E, 77 (2008) 041118.

[21] Johal R. S., Phys. Rev. E, 80 (2009) 041119.

[22] Rutten R., Esposito M. and Cleuren B., Phys. Rev. $B, 80$ (2009) 235112.

[23] Zhou Y. and Segal D., Phys. Rev. E, 82 (2010) 011120.

[24] Schmiedl T. and Seifert U., Europhys. Lett., 81 (2008) 20003.

[25] Tu Z. C., J. Phys. A, 41 (2008) 312003.

[26] Esposito M., Lindenberg K. and Van den Broeck C., Europhys. Lett., 85 (2009) 60010.

[27] Esposito M., Lindenberg K. and Van den Broeck C., Phys. Rev. Lett., 102 (2009) 130602.

[28] Izumida Y. and Okuda K., Europhys. Lett., 83 (2008) 60003.

[29] Izumida Y. and Okuda K., Prog. Theor. Phys. Suppl., 178 (2009) 163-168.

[30] Izumida Y. and Okuda K., Phys. Rev. E, 80 (2009) 021121.

[31] Izumida Y. and Okuda K., Eur. Phys. J. B, 77 (2010) 499.

[32] Gaveau B., Moreau M. and Schulman L. S., Phys. Rev. Lett., 105 (2010) 060601.

[33] Moreau M., Gaveau B. and Schulman L. S., Eur. Phys. J. D, 62 (2011) 67.

[34] Esposito M., Kawai R., Lindenberg K. and Van den Broeck C., Phys. Rev. E, 81 (2010) 041106.

[35] Esposito M., Kawai R., Lindenberg K. and Van Den Broeck C., Phys. Rev. Lett., 105 (2010) 150603.

[36] Benenti G., Saito K. and Casati G., Phys. Rev. Lett.,
106 (2011) 230602

[37] Seifert U., Phys. Rev. Lett., 106 (2011) 020601.

[38] Onsager L., Phys. Rev., 37 (1931) 405.

[39] De Groot S. R. and Mazur P., Non-equilibrium Thermodynamics (Dover, New York) 1984.

[40] Chen L. and Yan Z., J. Chem. Phys., 90 (1989) 3740.

[41] Velasco S., Roco J. M. M., Medina A. and Calvo Hernández A., J. Phys. D, 34 (2001) 1000.

[42] Izumida Y., Doctoral Thesis (Hokkaido University) 2011.

[43] Van den Broeck C., Kawai R. and Meurs P., Phys. Rev. Lett., 93 (2004) 090601.

[44] Van den Broeck C. and Kawai R., Phys. Rev. Lett., 96 (2006) 210601.

[45] van den Broek M. and Van den Broeck C., Phys. Rev. Lett., 100 (2008) 130601. 